\title{
Polygenic scores for UK Biobank scale data
}

Timothy Shin Heng Mak ${ }^{1}$, Robert Milan Porsch ${ }^{1}$, Shing Wan Choi ${ }^{2}$, Pak Chung Sham ${ }^{1,3,4^{*}}$

1 Centre for Genomic Sciences, University of Hong Kong, Hong Kong, China

2 MRC Social, Genetic and Developmental Psychiatry Centre, Institute of Psychiatry, King's College

London, London, United Kingdom

3 Department of Psychiatry, University of Hong Kong, Hong Kong, China

4 State Key Laboratory of Brain and Cognitive Sciences, University of Hong Kong, Hong Kong, China

* Corresponding author: Pak Chung Sham (pcsham@hku.hk)

\section{Abstract}

Polygenic scores (PGS) are estimated scores representing the genetic tendency of an individual for a disease or trait and have become an indispensible tool in a variety of analyses. Typically they are linear combination of the genotypes of a large number of SNPs, with the weights calculated from an external source, such as summary statistics from large meta-analyses. Recently cohorts with genetic data have become very large, such that it would be a waste if the raw data were not made use of in constructing PGS. Making use of raw data in calculating PGS, however, presents us with problems of overfitting. Here we discuss the essence of overfitting as applied in PGS calculations and highlight the difference between overfitting due to the overlap between the target and the discovery data (OTD), and overfitting due to the overlap between the target the the validation data (OTV). We propose two methods - cross prediction and split validation - to overcome OTD and OTV respectively. Using these two methods, PGS can be calculated using raw data without overfitting. We show that PGSs thus calculated have better predictive power than those using summary statistics alone for six phenotypes in the UK Biobank data.

\section{Introduction}

Polygenic scores, or polygenic risk scores (PGS), have become an indispensible tool in genetic studies 16 $[1,2,3,4,5,6,7,8,9,10,11,12,13,14]$. Polygenic scores are routinely calculated in small and large 17 cohorts with genotype data, and they represent individual genetic tendencies for particular traits or 18 
diseases. As such they can be used for stratifying individuals into different risk groups based on their 19 genetic makeup [14, 3, 4, 15]. Potentially, different interventions could be given to individuals with 20 different risks, which is part of the vision in personalized medicine $[16,17]$.

Currently, however, the predictive ability of PGS for complex traits remains considerably lower than the maximum possible given their heritability, although with increasing sample sizes and the number of Genome-wide association studies, the power is set to increase [18, 19, 12]. Nonetheless, even before the objective of personalized medicine can be achieved, PGS can be used for studying the genetic influence of different phenotypes. By examining the correlation between PGS and various phenotypes, researchers can gather evidence for whether the genetic influence on certain traits were pleiotropic or specific $[20,21,22,23,11,6,7]$. For example, using PGS, Power et al[11] showed that genetic tendency for schizophrenia and bipolar disorder were predictive of creativity, supporting earlier suggestions that creativity and tendency towards major psychotic illnesses may share some common roots.

Polygenic scores are calculated as weighted sums of the genotypes, with weights typically derived from large cohorts or meta-analyses. A key requirement in the calculation of PGS is that the same individuals be not used both in the calculation of the weights (in the discovery dataset) and the PGS (in the target dataset). Indeed, in general, samples in the discovery and target dataset should not even be related [24]. Overlap or relatedness between the samples is expected to lead to overfitting, i.e. the inflation in measures of the fit in the target dataset.

Recently, cohorts with genotype data have become very large. Examples of such cohorts include the UK Biobank[25] ( $n \approx 500,000)$, the 23andMe cohort [26] $(n \approx 600,000)$, and the deCode cohort [27] $(n \approx 350,000)$. In studies to date using the UK Biobank, for example, following the recommended practice, weights for the PGS were calculated from summary statistics and data external to the cohort $[13,28,27]$. Although sensible as a measure to avoid overfitting, the exclusion of the target dataset from the calculation of the summary statistics in these cases can be wasteful, given that such large sample sizes are involved.

In this paper, we show that it is possible to calculate PGS using the target dataset while avoiding overfitting, which can lead to higher predictive power than PGS calculated from summary statistics alone.

\section{Results}

As an illustration of the potential gain in power using the target dataset in the calculation of PGS, 48 consider the correlation between the phenotype and the PGS calculated using the method of this paper, 49 which we call cross prediction, compared to using summary statistics only, as presented in Figure $1 . \quad 50$ The comparison is made using a cohort of 353,465 white British participants in the UK Biobank study 51 [25]. We see that for all 6 phenotypes, using the data available in the UK Biobank alone gives a PGS 52 


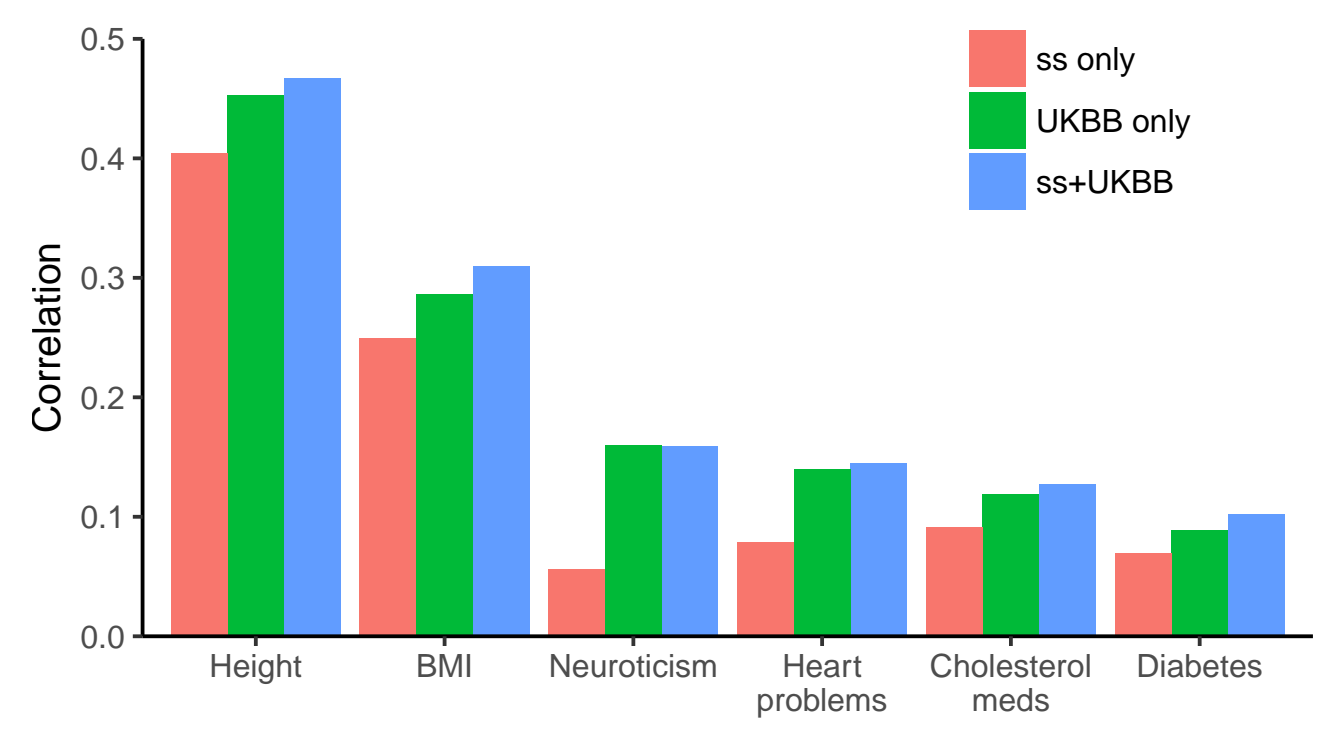

Figure 1: Correlation between phenotype and PGS calculated using summary statistics (ss) only, UKBB data only, and summary statistics plus UKBB data, in a cohort of 353,465 particpants in the UK Biobank.

with visibly higher correlation with the phenotype than the equivalent PGS calculated using summary 53 statistics. The correlation was even higher when the UKBB data was meta-analysed with the summary 54 statistics. In this section, we introduce the methods used in calculating the PGS in Figure 1 . We show 55 how these methods avoid overfitting and thus the improvement seen in Figure 1 is due to genuine increase 56 in power because of the data available in the UK Biobank. We defer the details of the simulations to ${ }_{57}$ the Methods section at the end of the article.

\section{Three types of overfitting in calculating polygenic scores}

In their review article, Wray et al[24] pointed out that if the same individuals were used in both the 60 target dataset and the discovery dataset or if they were related, estimates of the predictive power of PGS would be inflated. Although not specifically mentioned, the phenomenon underlying this was that of overfitting of the data to the target dataset. Here, we define overfitting to be the inflation of the correlation of the PGS with the genetic component in the target dataset over a completely independent (unseen) external dataset. More precisely, let us assume the following linear model 


$$
\begin{aligned}
& \boldsymbol{y}=\boldsymbol{X} \boldsymbol{\beta}+\boldsymbol{\epsilon} \\
& \boldsymbol{\epsilon} \sim N\left(\mathbf{0}, \sigma^{2} \boldsymbol{I}\right)
\end{aligned}
$$

where $\boldsymbol{y}=\left(y_{1}, y_{2}, \ldots, y_{n}\right)^{\prime}$ denotes a vector of phenotype from $n$ independent individuals from the target 66 dataset. Let $\boldsymbol{X} \boldsymbol{\beta}$ denote the genetic component and $\boldsymbol{\epsilon}=\left(\epsilon_{1}, \epsilon_{2}, \ldots, \epsilon_{n}\right)^{\prime}$ residual environmental effects, 67 with $\epsilon_{i}$ assumed independently and identically distributed. We assume $\boldsymbol{X}=\left(\boldsymbol{x}_{1}^{\prime}, \boldsymbol{x}_{2}^{\prime}, \ldots, \boldsymbol{x}_{n}^{\prime}\right)^{\prime}$ is a $n$-by- $p \quad{ }_{68}$ genotype matrix and $\boldsymbol{\beta}$ a vector of causal effects. In the case where adjustment for principal components ${ }_{69}$ is necessary[29], we assume that both $\boldsymbol{y}$ and $\boldsymbol{X}$ have the principal components of $\boldsymbol{X}$ regressed out of 70 them. A PGS for an individual $i$ is an estimate of $\boldsymbol{x}_{i} \boldsymbol{\beta}$, denoted $\mathrm{PGS}_{i}=\boldsymbol{x}_{i} \hat{\boldsymbol{\beta}}$. We define overfitting as ${ }_{71}$

$$
\operatorname{Cor}\left(\boldsymbol{x}_{i} \hat{\boldsymbol{\beta}}, y_{i}\right)>\operatorname{Cor}\left(\boldsymbol{x}_{i}^{E} \hat{\boldsymbol{\beta}}, y_{i}^{E}\right)
$$

where $\left(\boldsymbol{x}_{i}, y_{i}\right)$ is a randomly chosen sample from the target dataset, and $\left(\boldsymbol{x}_{i}^{E}, y_{i}^{E}\right)$ is a randomly chosen 72 sample from an independent external dataset. Given the independence of $\boldsymbol{X} \boldsymbol{\beta}$ and $\boldsymbol{\epsilon}$, equation (3) can 73 be expressed as

$$
\sqrt{h^{2}} \operatorname{Cor}\left(\boldsymbol{x}_{i} \hat{\boldsymbol{\beta}}, \boldsymbol{x}_{i} \boldsymbol{\beta}\right)+\sqrt{1-h^{2}} \operatorname{Cor}\left(\boldsymbol{x}_{i} \hat{\boldsymbol{\beta}}, \boldsymbol{\epsilon}_{i}\right)>\sqrt{h_{E}^{2}} \operatorname{Cor}\left(\boldsymbol{x}_{i}^{E} \hat{\boldsymbol{\beta}}, \boldsymbol{x}_{i}^{E} \boldsymbol{\beta}\right)+\sqrt{1-h_{E}^{2}} \operatorname{Cor}\left(\boldsymbol{x}_{i}^{E} \hat{\boldsymbol{\beta}}, \boldsymbol{\epsilon}_{i}^{E}\right)
$$

where $h^{2}=\frac{\operatorname{Var}\left(\boldsymbol{x}_{i} \boldsymbol{\beta}\right)}{\operatorname{Var}\left(y_{i}\right)}$ and $h_{E}^{2}=\frac{\operatorname{Var}\left(\boldsymbol{x}_{i}^{E} \boldsymbol{\beta}\right)}{\operatorname{Var}\left(y_{i}^{E}\right)}$ denote the heritability of the trait in the target and the external 75 dataset respectively, and $\operatorname{Cor}\left(\boldsymbol{x}_{i}^{E} \hat{\boldsymbol{\beta}}, \boldsymbol{\epsilon}_{i}^{E}\right)=0$ by definition. A sufficient condition for no overfitting is 76 thus

$$
\begin{aligned}
\operatorname{Cor}\left(\boldsymbol{x}_{i} \hat{\boldsymbol{\beta}}, \boldsymbol{x}_{i} \boldsymbol{\beta}\right) & =\operatorname{Cor}\left(\boldsymbol{x}_{i}^{E} \hat{\boldsymbol{\beta}} \boldsymbol{x}_{i}^{E} \boldsymbol{\beta}\right) \\
\operatorname{Cor}\left(\boldsymbol{x}_{i} \hat{\boldsymbol{\beta}}, \boldsymbol{\epsilon}_{i}\right) & =0 \\
h^{2} & =h_{E}^{2}
\end{aligned}
$$

The fact that when the target data is used to calculate the summary statistics $\hat{\boldsymbol{\beta}}$, overfitting occurs, 78 can be seen by considering a Directed Acyclic Graph (DAG), showing the relationship between $\boldsymbol{X} \hat{\boldsymbol{\beta}} \quad 79$ and $\boldsymbol{X} \boldsymbol{\beta}$ (Fig 2(a)). (A DAG can be seen as a graphical representation of the probabilistic dependency 80 of the different variables, and its interpretation is grounded in probability theory [30]. Two variables 81 are 'connected' if a line can be traced through the graph connecting the two variables, except when 82 a 'collider' is present along the path that connects the two. A 'collider' is a variable within a path 83 where the two edges connecting it are both arrows pointing towards it, such as the variables $\boldsymbol{y}, \hat{\boldsymbol{\beta}}, \quad 84$ 

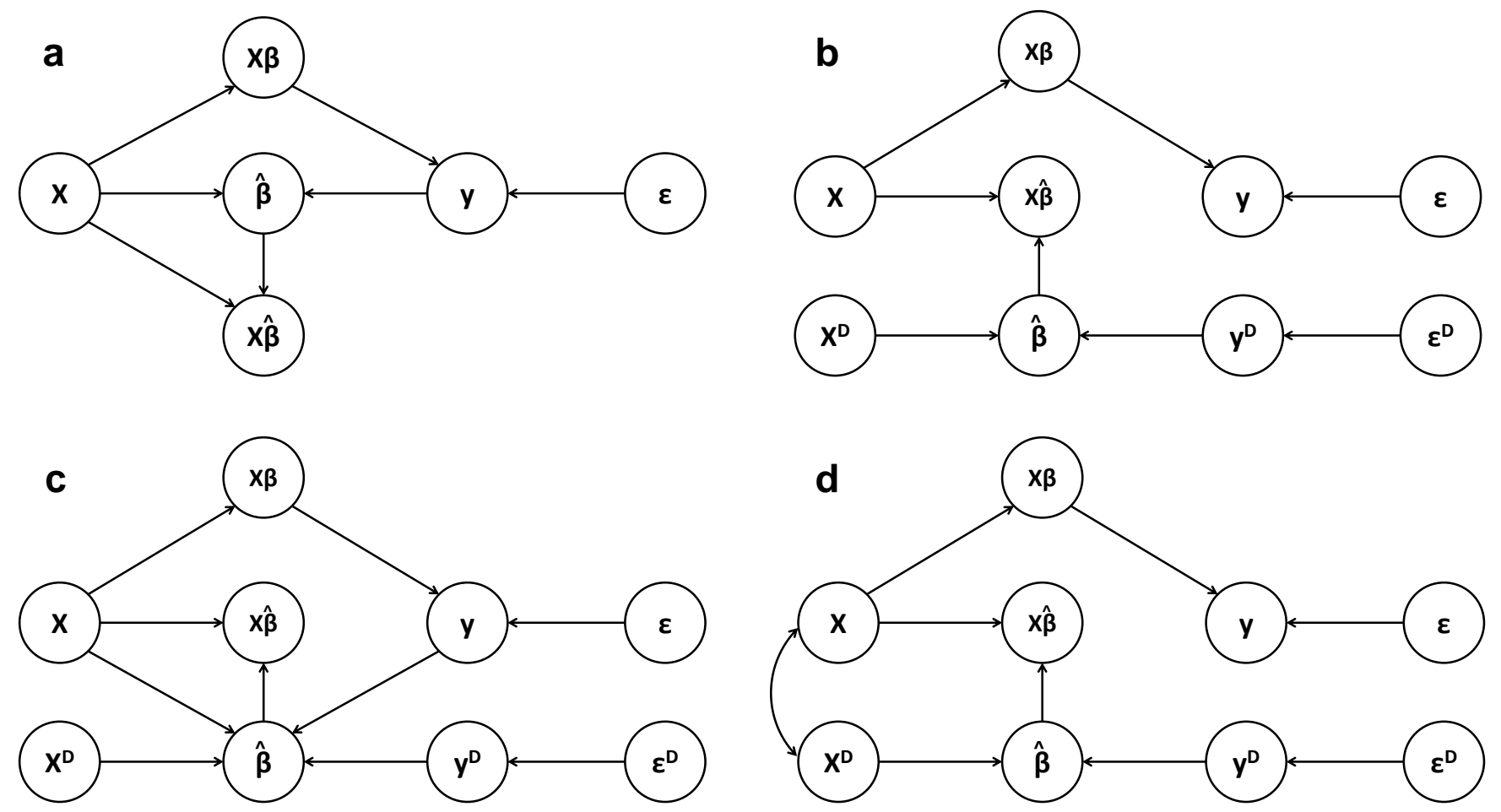

Figure 2: DAGs illustrating the relationship between the different variables in PGS estimation (a) when the target data is also used in the estimation of $\boldsymbol{\beta}$, (b) when a separate discovery dataset $\left(\boldsymbol{X}^{D}, \boldsymbol{y}^{D}\right)$ is used, (c) when the target dataset is used in choosing the tuning paramter or the best $\hat{\boldsymbol{\beta}}$ among a set of different $\hat{\boldsymbol{\beta}}_{\mathrm{s}}$, and $(\mathrm{d})$ when the target dataset is genetically related to the discovery dataset.

and $\boldsymbol{X} \hat{\boldsymbol{\beta}}$, in Fig 2(a). Probabilistically, variables that are connected are expected to be dependent and correlated. Variables that are not connected are not dependent and thus not correlated.) We see that $\boldsymbol{X} \hat{\boldsymbol{\beta}}$ is connected to $\boldsymbol{\epsilon}$ through $\boldsymbol{y}$ and thus expected to be correlated. Moreover, because in general we expect $\operatorname{Cor}\left(\boldsymbol{x}_{i} \hat{\boldsymbol{\beta}}, y_{i}\right)>0$ and $\operatorname{Cor}\left(y_{i}, \epsilon_{i}\right)>0$, we expect $\operatorname{Cor}\left(\boldsymbol{x}_{i} \hat{\boldsymbol{\beta}}, \epsilon_{i}\right)>0$, resulting in overfitting. In this article, we refer to this type of overfitting as OTD (Overfitting due to the overlap between the Target and the Discovery data).

In Fig 2(b) we see that if we use an external discovery dataset for estimating $\boldsymbol{\beta}, \operatorname{Cor}\left(\boldsymbol{x}_{i} \hat{\boldsymbol{\beta}}, \epsilon_{i}\right)=0$, because the path between $\hat{\boldsymbol{\beta}}$ and $\boldsymbol{y}$ is broken. Moreover, if the external discovery sample $\boldsymbol{x}^{D}, \boldsymbol{x}^{E}$, and $\boldsymbol{x}$ are all drawn from the same population, $\operatorname{Cor}\left(\boldsymbol{x}_{i} \hat{\boldsymbol{\beta}}, \boldsymbol{x}_{i} \boldsymbol{\beta}\right)=\operatorname{Cor}\left(\boldsymbol{x}_{i}^{E} \hat{\boldsymbol{\beta}}, \boldsymbol{x}_{i}^{E} \boldsymbol{\beta}\right)$ and overfitting is avoided.

A less appreciated kind of overfitting can be seen in Fig 2(c). Here, although the target dataset is 94 not used for estimating $\boldsymbol{\beta}$, it is used for choosing a $p$-value threshold in the construction of PGS, as 95 represented by the arrows pointing to $\hat{\boldsymbol{\beta}}$ from $\boldsymbol{X}$ and $\boldsymbol{y}$. The fact that we generally choose the $p$-value 96 threshold that maximizes the correlation between the PGS and the phenotype means that there is a 97 Winner's curse such that the apparent correlation between the PGS and the phenotype is higher than 98 it would be in an external dataset. In this article we refer to overfitting due to the target data being 
used in validation OTV (Overfitting due to the overlap between the Target and the Validation data). 100

Finally, let us note that the inflation of correlation as cautioned by Wray et al[24] concerns not only 101 the overlapping of samples. Rather, Wray et al[24] pointed out that inflation of correlation was likely if 102 the target dataset were genetically related to the discovery dataset. We illustrate this situation in Fig 103 2(d), where correlations are expected between $\boldsymbol{x}$ and $\boldsymbol{x}^{D}$. Here, although we still have $\operatorname{Cor}\left(\boldsymbol{x}_{i} \hat{\boldsymbol{\beta}}, \epsilon_{i}\right)={ }_{104}$ 0, we cannot expect $\operatorname{Cor}\left(\boldsymbol{x}_{i} \hat{\boldsymbol{\beta}}, \boldsymbol{x}_{i} \boldsymbol{\beta}\right)=\operatorname{Cor}\left(\boldsymbol{x}_{i}^{E} \hat{\boldsymbol{\beta}}, \boldsymbol{x}_{i}^{E} \boldsymbol{\beta}\right)$, leading to overfitting. For the purposes of 105 constructing PGS in large cohorts, however, this type of overfitting is of arguably less importance, 106 since we are not interested in some external population $\boldsymbol{x}^{E}$. In any case, accounting for differences in 107 relatedness between the sample population and the general population at large is difficult and beyond 108 the scope of this paper.

\section{Cross-prediction as a method to overcome overfitting due to the overlap of ${ }_{110}$ the target with the discovery data

As already noted above, overfitting can be avoided by breaking the path connecting $\boldsymbol{y}$ to $\hat{\boldsymbol{\beta}}$. One way 112 to do this in practice is to use an independent discovery dataset for estimating $\boldsymbol{\beta}$ (Fig 2(b)). When 113 faced with a large target dataset which we also want to use as our discovery dataset, we can repeat this 114 procedure in a cross-validation-like manner, i.e. we split the data into a number of folds, and repeatedly 115 estimate $\boldsymbol{X} \hat{\boldsymbol{\beta}}$ for the different folds, using the remaining folds for discovery. We call this procedure cross- $\quad{ }_{116}$ prediction (Figure 3(a)), to distinguish it from the more familiar procedure of cross-validation where ${ }_{117}$ fold-splitting is used only for choosing tuning parameters [31, 32, 33]. If external summary statistics are 118 available, these can also be meta-analysed with those calculated from the discovery folds. To combine 119 the PGS calculated in the different folds, we standardize them before stacking them together to form 120 the final PGS. Standarizing and stacking them in this way will imply that the resulting stacked PGS ${ }_{121}$ represents the average correlation between the particular variable and the fold-specific PGS. Moreover, 122 we prove that stacking the fold-specific PGS in this way preserves independence between individual ${ }_{123}$ elements of $\boldsymbol{X} \hat{\boldsymbol{\beta}}$ and $\boldsymbol{\epsilon}$ and therefore does not introduce overfitting. Both of these proofs are presented ${ }_{124}$ in the Methods section.

\section{Split-validation as a method to overcome overfitting due to the overlap of ${ }_{126}$ the target with the validation data

In practical application of PGS, we do not simply have one PGS. Far more often, PGS are calculated for

a range of $p$-value thresholds and the best one chosen. Letting $\hat{\mathbf{B}}$ denote a matrix of coefficients where 128 each column represent a vector of $\hat{\boldsymbol{\beta}}$ with different elements set to zero for different $p$-value thresholds, ${ }_{130}$ our estimated PGS is a matrix $\hat{\boldsymbol{Z}}=\boldsymbol{X} \hat{\mathbf{B}}$ rather than a vector. This applies to each of the folds in cross- ${ }_{131}$ 


\section{a}

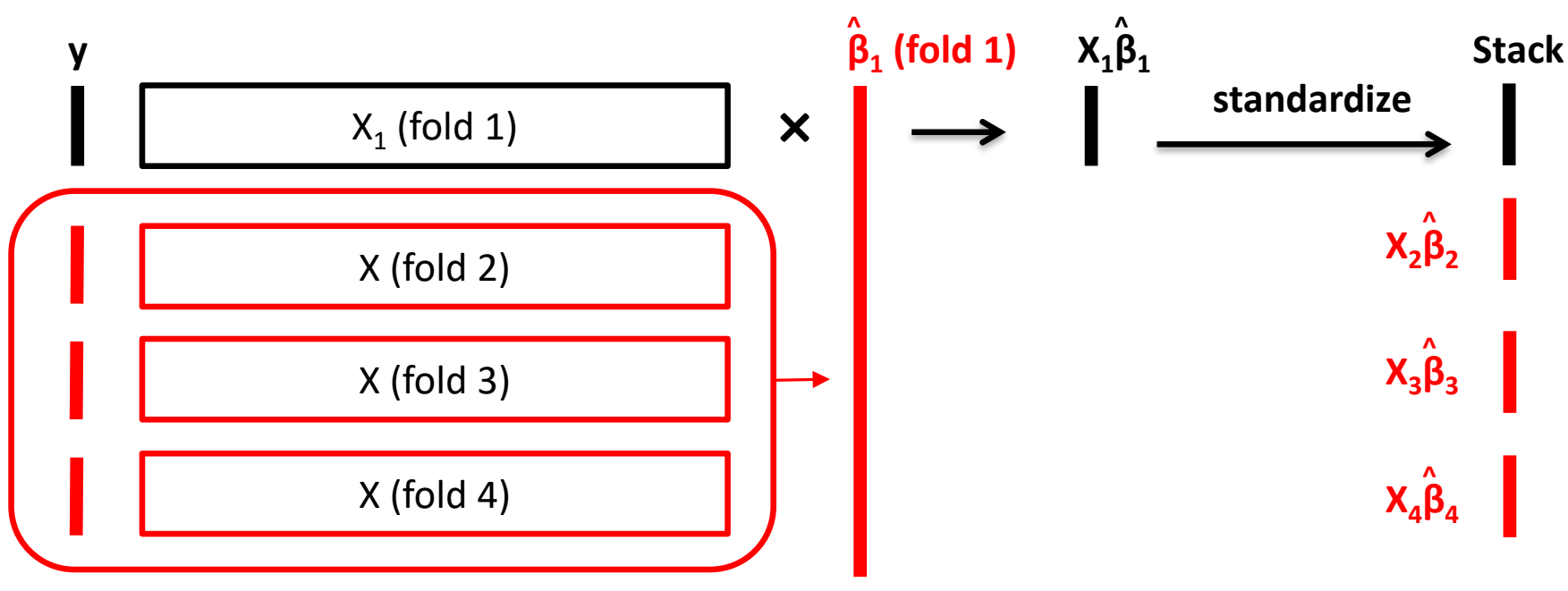

b

SNP $\rightarrow \quad \lambda \rightarrow$

Sample
$\downarrow$

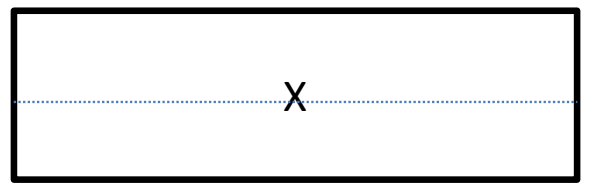

$\hat{\mathrm{B}}$

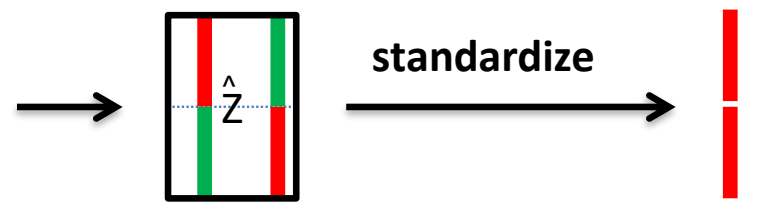

Figure 3: (a) Cross-prediction. The data $(\boldsymbol{X}, \boldsymbol{y})$ is split into 4 folds. For fold 1, the coefficients $\hat{\boldsymbol{\beta}}_{1}$ is estimated from folds 2,3, and 4. The estimated PGS $\boldsymbol{X}_{1} \hat{\boldsymbol{\beta}}_{1}$ is standardized and stacked together to form the final PGS. (b) Split-validation. Let $\boldsymbol{X}$ deonte the genotype matrix, $\hat{\mathbf{B}}$ the matrix of coefficients, and $\lambda$ indices the $p$-value threshold. Let $\boldsymbol{Z}=\boldsymbol{X}_{k} \hat{\mathbf{B}}_{-k}$ be the matrix of PGS calculated for the $k^{\text {th }}$ fold. $\hat{\boldsymbol{Z}}$ and $\boldsymbol{X}_{k}$ are split into two halves. The green columns are the columns of $\hat{\mathbf{B}}$ corresponding to the $p$-value threshold selected by validation. The red columns are the corresponding $\hat{\boldsymbol{Z}}$ taken to form the PGS. 


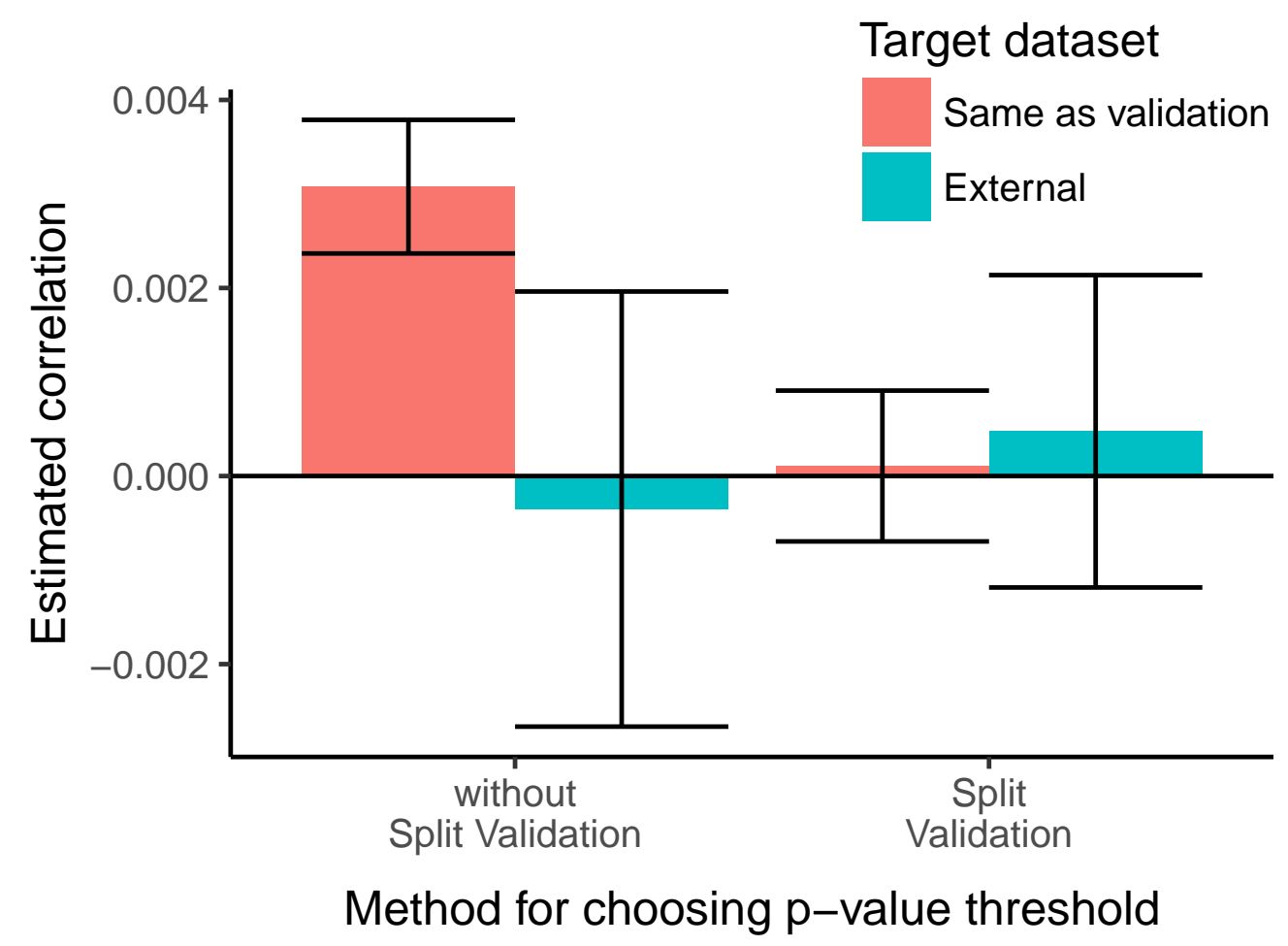

Figure 4: Barplots of mean estimated correlations between simulated null phenotypes (no genetic component) and the estimated PGSs, when the target dataset doubles up as the validation dataset, and when the target dataset is external. Error bars represent $95 \%$ confidence intervals.

prediction, where for each fold $k$ we have a matrix of standardized PGS $\hat{\boldsymbol{Z}}_{k}$. We need to choose the best 132 column of $\hat{\boldsymbol{Z}}_{k}$ for each fold in order to form the final PGS, a step we refer to as validation. A common ${ }_{133}$ practice in PGS construction is to double up the target dataset as the validation dataset $[13,10,34]$. If 134 put in the context of cross-prediction, this translates to performing validation and calculating the PGS 135 within the target fold using the same data. However, as mentioned above, this can lead to overfitting, 136 in particular OTV. Although the impact of this type of overfitting is commonly believed to be small, 137 we illustrate its impact in the UK Biobank dataset by results from a simulation, whose details are 138 given in the Methods section. Figure 4 shows the estimated correlations between multiple randomly 139 generated (null) phenotypes and their PGSs calculated using cross-prediction. We see that although 140 the phenotype was generated with no genetic component, when the target data doubled up as the 141 validation data, the estimated correlations were inflated, compared to correlations with the phenotype 142 in an external dataset. In Figure 5, we show this bias in terms of inflation in Type 1 error. When 143 $p$-values between the estimated PGS and the phenotype are plotted against the expected distribution, 144 there is a small but visible inflation in the statistics. Our strategy to overcome this is split-validation. 145 In both Figures 4 and 5, we see that the method of split validation did not incur overfitting. 146

The idea of split validation is similar to that of cross prediction. We first split the target dataset 147 (or the target fold in cross-prediction) into two halves. We take turn to use each half for validation 148 


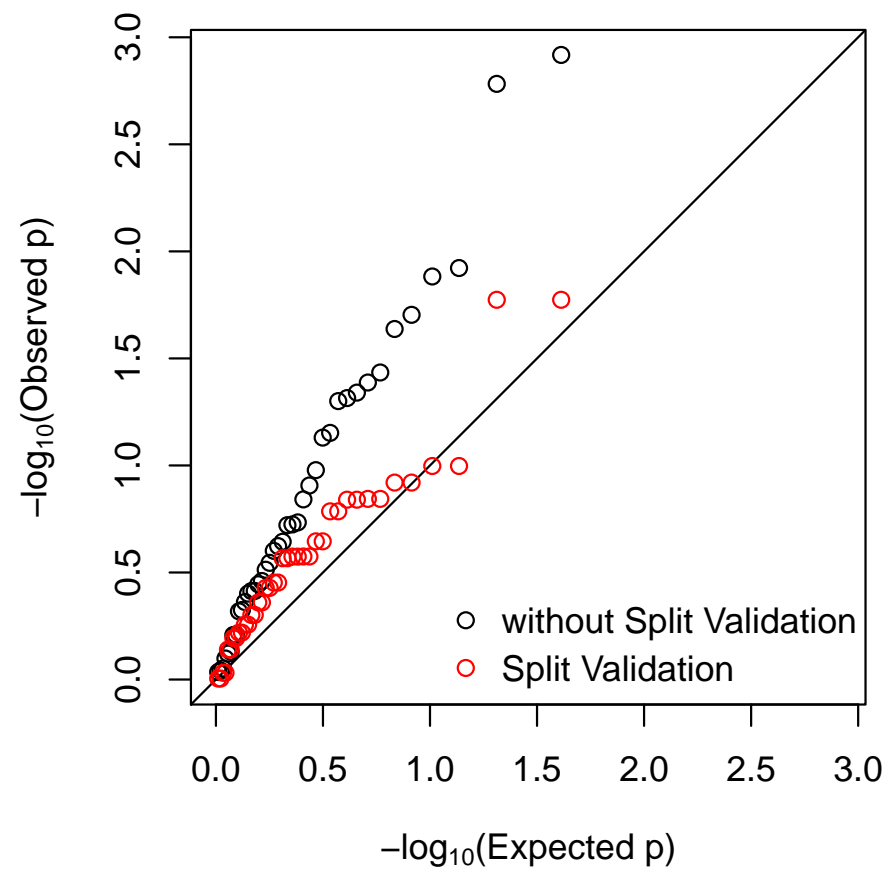

Figure 5: qq plot of $p$-values calculated for the relationship between the PGS and phenotype when the target data is also used for validation under a simulated null model. 


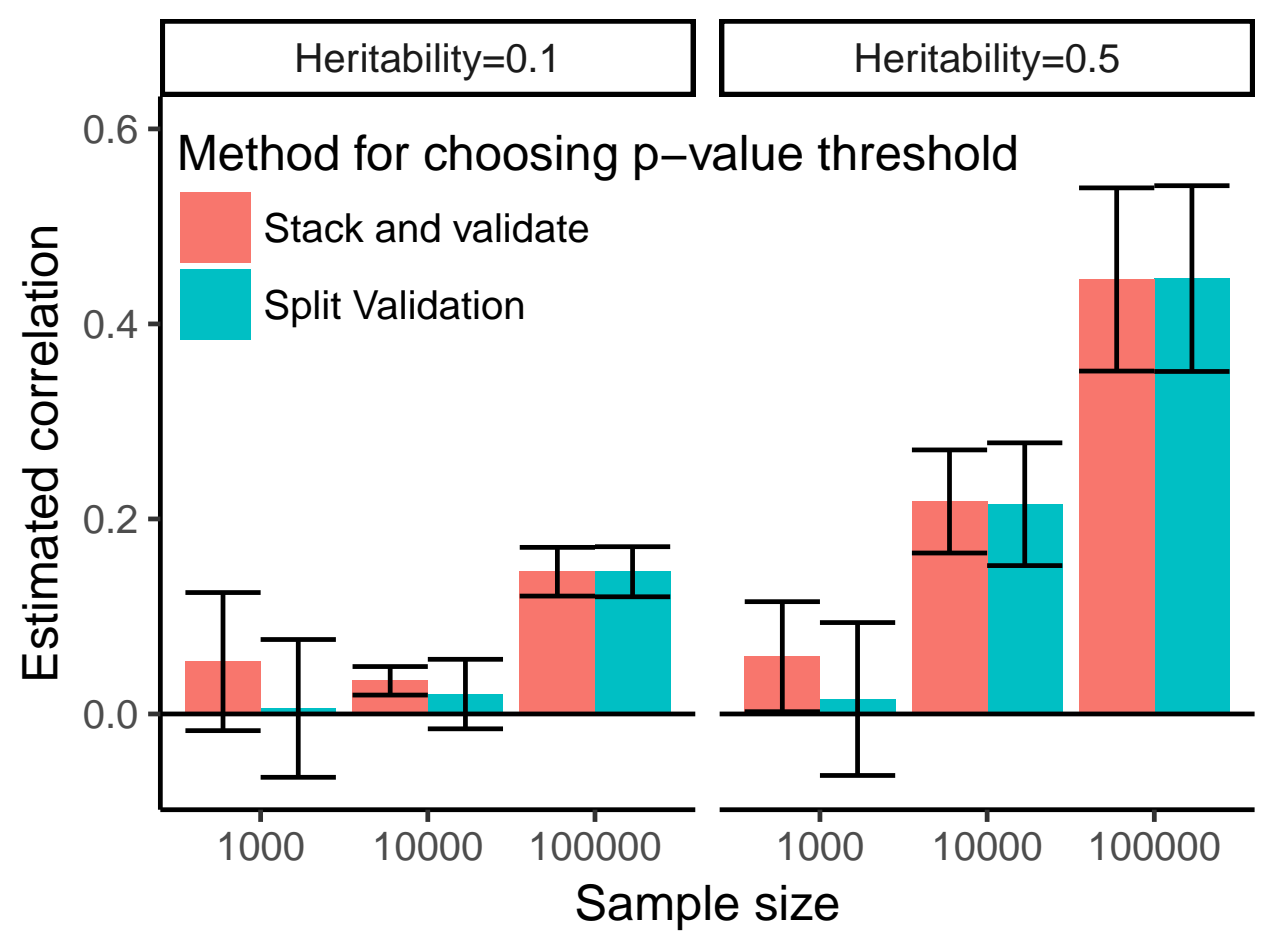

Figure 6: Comparison of split-validation vs "stack and validate" in calculating PGS. Mean and standard deviation of estimated correlation from 10 simulated phenotypes are plotted for each scenario.

(i.e. the selection of the $p$-value threshold), and calculate the PGS in the other half using the $p$-value ${ }_{149}$ threshold selected in the other half and weights derived in the discovery dataset. A diagram illustrating 150 split-validation is given in Figure 3(b).

\section{Sample size concerns}

One possible concern with the cross-prediction + split-validation strategy is that instead of carrying 153 out validation once, we carry out validation in multiple sub-samples within the target dataset, and this may reduce the power in choosing the best $p$-value threshold because of smaller samples. An alternative method that does not prevent OTV (but does prevent OTD) is to stack up the (standardized) PGS ( $\left.\hat{\boldsymbol{Z}}_{k}\right)$ first (calculated for all $p$-value thresholds), and then validate them against the phenotype to choose the best $p$-value threshold. In Figure 6, we compare the performance of cross-prediction + split-validation vs the latter strategy (stack and validate) using sub-samples of the UK Biobank data and simulated phenotypes under two heritability scenarios $\left(h^{2}=0.1\right.$ and 0.5). 2,000 SNPs among 734,447 SNPs were assigned to be causal. It can be seen that when the sample size was 100,000, basically there were no difference in the predictive power of the PGS calculated using split-validation and stack and validate. When the sample size was smaller, we see that the predictive power of split-validation was reduced, particularly in the heritability $=0.1$ setting.

\section{4} 155 156 157 158 159 160 161 162 163 164 


\section{Discussion}

In this article, we show how cross-prediction, combined with split-validation, can be used to calculate 166 PGS in large cohorts such as the UK Biobank. This can lead to a considerable increase in predictive power compared to using summary statistics alone. An overview of what constitutes overfitting is also given and it is shown that cross-prediction combined with split-validation overcomes both overfitting due to the target dataset overlapping with the discovery dataset (OTD) and with the validation dataset (OTV). The basic principle of the approach is the separation of the discovery, the validation, and the target subset of the dataset, and the combination of the resulting PGS from the different subsets through standardizing and stacking, which is shown preserve predicitve power and independence between the subsets.

One possible issue with this appraoch is that performing validation in different subsets and stacking the resulting PGS can reduce predictive power, compared to using the same data both for validation and prediction. However, it may be argued that with sample sizes of the magnitude of the UK Biobank, this is not an important issue.

In this article, we have not discussed overfitting due to other kinds of overfitting. In particular, we have not discussed possible overfitting due to the sample being related. Indeed it has been pointed out that the UK Biobank consists of a considerable number of second and third degree relatives [25]. This can lead to inflated estimates of the predictive accuracy of the PGS if estimates of $r^{2}$ from the UK Biobank were extrapolated to the general population. On the other hand, we note that if our aim is to assess genetic correlation within the UK Biobank sample, then this type of overfitting is not relevant.

Usually genetic correlations can be assessed by examining the relationship between the PGS and various phenotypes. An important point to note is that overfitting can still occur when correlating different PGS calculated using the method of this paper. This is because in cross-prediction we try to keep the discovery and the target samples separate. However, when two PGS are both calculated using cross-prediction, their discovery samples can overlap, leading to overfitting.

We conclude with a number of suggestions for future work. First, depending on the number of folds use, a proportion of the sample is left out in the calculation of the summary statistics. It is unsure whether there can be a procedure that uses all data and also avoids OTD and OTV. Secondly, the current procedure is stochastic as the folds are randomly defined. The resulting PGS is also not a linear predictor in that it is not calculated as a linear combination of $\boldsymbol{X}$. Rather it is a mixture of different linear combinations. This has the disadvantage that theoretical properties of the PGS are less easily obtained. In principle, it is possible to find estimates of $\boldsymbol{\beta}$ such that when multiplied with $\boldsymbol{X}$, equals the CP PGS as calculated in our study. However, in our preliminary simulations, these estimates of $\boldsymbol{\beta}$ had very poor performance in external validation and we have not pursued this approach further. It is also possible in principle to extend this work further to the case where the number of folds used equals 
the sample size, such that we have a jackknife-like procedure for cross-prediction. This approach has 200 not been studied. Thirdly, calculation of PGS using cross-prediction is currently time consuming for 201 large cohorts and a large number of SNPs. In the simulations we have limited the number of SNPs to 202 around 700,000. It may be possible to perform cross-prediction on a pre-selected set of SNPs using for 203 example, informed pruning (clumping) [35]. However, if this set of SNPs were selected based on the 204 entire dataset, overfitting would also arise, and future work is needed to minimize or avoid this bias. 205

\section{Methods}

\section{PGS for six UK Biobank phenotypes}

353,465 white British participants from the UK Biobank study were selected for these analyses. We used the genotype array of 734,447 SNPs, made available by the UK Biobank. The 6 phenotypes considered were height $(\mathrm{ID}=50)$, BMI $(\mathrm{ID}=21001)$, neuroticism $(\mathrm{ID}=20127)$, heart problems, taking medication for lowering cholesterol (ID=6153, 6177), and diabetes ( $\mathrm{ID}=2443)$. Where multiple measurements were taken, the average was used. The variable "heart problems" was defined as a score from 0 to 3 based on the question "Vascular/heart problems diagnosed by doctor" (ID=6150), where 3 represents "Heart attack" or "Stroke", 2 represents "Angina", 1 represents "High blood pressure", and 0 "None of the above". The corresponding summary statistics were taken from the following studies: height[36], BMI[37], Neuroticism[38], Heart problems[39] (summary statistics for coronary artery disease), Medication for lowering cholesterol[40] (summary statistics for total cholesterol levels), Diabetes[41]. Only variants that were present in both the summary statistics and the UK Biobank genotype array were used for constructing PGS, both for the summary-statistics derived PGS and for cross-prediction. 5fold cross-prediction was used with split-validation. All analyses, including the correlation between the phenotype and the PGS, were adjusted for the first 20 principal components and inferred gender. For Figure 1, selection of the $p$-value threshold for the summary-statistics only PGS was performed on an independent sample of 10,000 white British participants from the UK Biobank.

\section{Simulated phenotypes from the UK Biobank}

For Figures 4 and 5, the same cohort of 353,465 white British participants from the UK Biobank was used. $\quad 225$ The phenotype was a simulated vector of $353,465 N(0,1)$ random variables and thus was completely ${ }^{226}$ independent of the genetic data. 5-fold cross-prediction was applied to compute the PGS. In the "without 227 Split-Validation" scenario, the method of "stack and validate" was used (see Results section). The 228 simulation was repeated 10 times and fold-specific correlations and $p$-values were plotted in the figures. 229 The "external" target dataset was an independent dataset of 10,000 white British participants randomly 230 selected from the UK biobank. No covariate adjustments were performed with these analyses. 
For Figure 6, the linear model of (1) was used to generate the phenotype, with heritability, i.e. 232 $\hat{\operatorname{Var}}(\boldsymbol{X} \boldsymbol{\beta}) /\left(\hat{\operatorname{Var}}(\boldsymbol{X} \boldsymbol{\beta})+\sigma^{2}\right)$ constrained to be either 0.1 or 0.5 . Samples of size 1,000, 10,000, and 233 100,000 were randomly selected from the 353,465 white British participants.

\section{Details of PGS calculation}

In all calculation of PGS in this paper, clumping and thresholding was used. First, summary statis- ${ }^{236}$ tics were clumped using the default settings in PLINK 1.9[42], where variants with an $R^{2}$ of 0.2 or ${ }^{237}$ above within a $250 \mathrm{~kb}$ region were "clumped" with the most significant SNP. $p$-value thresholds of ${ }_{238}$ $1 e^{-20}, 5 e^{-20}, 1 e^{-19}, 5 e^{-19}, \ldots, 0.001,0.005,0.01,0.02,0.03, \ldots, 0.99,1$ were used. Clumping and $p$-value 239 thresholding was performed independently for each fold in cross-prediction.

\section{Computation}

An R package (crosspred) has been written to perform cross-prediction and split-validation, and is avail- ${ }^{242}$ able on https://github.com/tshmak/crosspred/blob/master/CrossPrediction.md. The package is ${ }^{243}$ designed to be a wrapper around the package lassosum [43]. Although clumping and $p$-value thresh- ${ }^{244}$ olding was used throughout this paper to calculate PGS (as it is the more widely used method), in 245 principle, it is possible and even preferable to use lassosum instead, which can lead to better predictive ${ }^{246}$ power.

\section{Proof: standardizing PGS within fold before stacking approximates the ${ }_{248}$ average correlation of the PGS with another variable

Let $\boldsymbol{x}=\left(\boldsymbol{x}_{1}^{\prime}, \boldsymbol{x}_{2}^{\prime}, \ldots, \boldsymbol{x}_{N}^{\prime}\right)^{\prime}$ denote a stacked column of PGS, and $\boldsymbol{y}$ a column of phenotype. Further ${ }^{250}$ assume $\boldsymbol{x}$ is standardized within fold, such that $\mathbf{1}^{\prime} \boldsymbol{x}_{k}=\mathbf{0}$ and $\boldsymbol{x}_{k}^{\prime} \boldsymbol{x}_{k}=n_{k}$, and that $\boldsymbol{y}$ is standardized 251 such that $\mathbf{1}^{\prime} \boldsymbol{y}=\mathbf{0}$ and $\boldsymbol{y}^{\prime} \boldsymbol{y}=n=\sum_{k} n_{k}$ without loss of generality. The correlation of $\boldsymbol{x}$ with $\boldsymbol{y}$ is ${ }^{252}$ $\boldsymbol{x}^{\prime} \boldsymbol{y} / n$. Let the standard deviation of $\boldsymbol{y}$ within fold $k$ be $1 / s_{k}$. We have

$$
\frac{\boldsymbol{x}^{\prime} \boldsymbol{y}}{n}=\sum_{k} \frac{\boldsymbol{x}_{k}^{\prime} \boldsymbol{y}_{k} s_{k}}{n_{k}} \frac{n_{k}}{s_{k} n}
$$

where $\frac{\boldsymbol{x}_{k}^{\prime} \boldsymbol{y}_{k} s_{k}}{n_{k}}$ is the fold-specific correlation. Thus, $\frac{\boldsymbol{x}^{\prime} \boldsymbol{y}}{n}$ is a weighted average of the fold-specific correlation ${ }^{254}$ with weights $\frac{n_{k}}{s_{k} n}$. In general $s_{k}$ approximates 1 , such that the weights are approximately optimal. 255 


\section{Proof that $X \hat{\boldsymbol{\beta}}$ remain independent of with $\epsilon$ after stacking}

As in the main text, we assume that $\boldsymbol{X}=\left(\boldsymbol{X}_{1}^{\prime}, \boldsymbol{X}_{2}^{\prime}, \ldots, \boldsymbol{X}_{N}^{\prime}\right)^{\prime}, \boldsymbol{y}=\left(\boldsymbol{y}_{1}^{\prime}, \boldsymbol{y}_{2}^{\prime}, \ldots, \boldsymbol{y}_{N}^{\prime}\right)^{\prime}, \boldsymbol{\epsilon}=\left(\boldsymbol{\epsilon}_{1}^{\prime}, \boldsymbol{\epsilon}_{2}^{\prime}, \ldots, \boldsymbol{\epsilon}_{N}^{\prime}\right)^{\prime} .257$ Denote $\boldsymbol{z}_{k}=\boldsymbol{X}_{k} \hat{\boldsymbol{\beta}}$. From Figure 2(b), we establish that $\boldsymbol{z}_{k}$ is independent of $\boldsymbol{\epsilon}$ if $\hat{\boldsymbol{\beta}}$ is derived from a 258 different fold from $\boldsymbol{z}_{k}$. It follows that the $i^{\text {th }}$ element of $\boldsymbol{z}_{k}$, denoted $z_{k i}$ is independent of the $i^{\text {th }}$ element ${ }^{259}$ of $\boldsymbol{\epsilon}$, within a particular fold $\mathcal{F}$. In notation:

$$
f_{z_{i}, \epsilon_{i} \mid \mathcal{F}}\left(z_{i}, \epsilon_{i}\right)=f_{z_{i} \mid \mathcal{F}}\left(z_{i}\right) f_{\epsilon_{i} \mid \mathcal{F}}\left(\epsilon_{i}\right)
$$

Proof: $f_{z_{i}, \epsilon_{i}}\left(z_{i}, \epsilon_{i}\right)=f_{\epsilon_{i}}\left(\epsilon_{i}\right) f_{z_{i}}\left(z_{i}\right)$.

$$
\begin{aligned}
f_{z_{i}, \epsilon_{i}}\left(z_{i}, \epsilon_{i}\right) & =\sum_{\mathcal{F}} p(\mathcal{F}) f_{z_{i}, \epsilon_{i} \mid \mathcal{F}}\left(z_{i}, \epsilon_{i}\right) \\
& =\sum_{\mathcal{F}} p(\mathcal{F}) f_{z_{i} \mid \mathcal{F}}\left(z_{i}\right) f_{\epsilon_{i} \mid \mathcal{F}}\left(\epsilon_{i}\right)
\end{aligned}
$$

Now, because $\epsilon_{i}$ are assumed i.i.d. regardless of fold, we have

$$
\begin{aligned}
f_{\epsilon_{i} \mid \mathcal{F}}\left(\epsilon_{i}\right) & =f_{\epsilon_{i}}\left(\epsilon_{i}\right) \\
f_{z_{i}, \epsilon_{i}}\left(z_{i}, \epsilon_{i}\right) & =f_{\epsilon_{i}}\left(\epsilon_{i}\right) \sum_{\mathcal{F}} p(\mathcal{F}) f_{z_{i} \mid \mathcal{F}}\left(z_{i}\right) \\
& =f_{\epsilon_{i}}\left(\epsilon_{i}\right) f_{z_{i}}\left(z_{i}\right)
\end{aligned}
$$

completing the proof.

\section{Acknowledgement}

This research has been conducted using the UK Biobank Resource (project ID 23079, 28732). 


\section{References}

[1] Purcell SM, Wray NR, Stone JL, Visscher PM, O’Donovan MC, Sullivan PF, et al. Com- ${ }^{265}$ mon polygenic variation contributes to risk of schizophrenia and bipolar disorder. Nature. 266 2009;460(7256):748-52. doi:10.1038/nature08185.

[2] Opherk C, Gonik M, Duering M, Malik R, Jouvent E, Hervé D, et al. Genome-wide 268 genotyping demonstrates a polygenic risk score associated with white matter hyperinten- 269 sity volume in CADASIL. Stroke; a journal of cerebral circulation. 2014;45(4):968-72. 270 doi:10.1161/STROKEAHA.113.004461.

[3] Stahl EA, Wegmann D, Trynka G, Gutierrez-Achury J, Do R, Voight BF, et al. Bayesian inference ${ }^{272}$ analyses of the polygenic architecture of rheumatoid arthritis. Nature genetics. 2012;44(5):483-9. 273 doi:10.1038/ng.2232.

[4] Agerbo E, Sullivan PF, Vilhjálmsson BJ, Pedersen CB, Mors O, Børglum AD, et al. Polygenic 275 Risk Score, Parental Socioeconomic Status, Family History of Psychiatric Disorders, and the 276 Risk for Schizophrenia: A Danish Population-Based Study and Meta-analysis. JAMA psychia- ${ }^{277}$ try. 2015;72(7):635-41. doi:10.1001/jamapsychiatry.2015.0346.

[5] Krapohl E, Patel H, Newhouse S, Curtis CJ, von Stumm S, Dale PS, et al. Multi-polygenic score ${ }^{279}$ approach to trait prediction. Molecular Psychiatry. 2017;(May):1-7. doi:10.1038/mp.2017.163. 280

[6] Krapohl E, Euesden J, Zabaneh D, Pingault JB, Rimfeld K, von Stumm S, et al. Phenome- ${ }^{281}$ wide analysis of genome-wide polygenic scores. Molecular Psychiatry. 2016;21(9):1188-1193. 282 doi:10.1038/mp.2015.126.

[7] Byrne EM, Carrillo-Roa T, Penninx BWJH, Sallis HM, Viktorin A, Chapman B, et al. Ap- 284 plying polygenic risk scores to postpartum depression. Archives of Women's Mental Health. 285 2014;17(6):519-528. doi:10.1007/s00737-014-0428-5.

[8] Marquez-Luna C, Consortium TSTD, Price AL. Multi-ethnic polygenic risk scores improve risk 287 prediction in diverse populations. bioRxiv. 2016; p. 051458. doi:10.1101/051458.

[9] Ruderfer DM, Fanous AH, Ripke S, McQuillin A, Amdur RL, Gejman PV, et al. Polygenic dissection 289 of diagnosis and clinical dimensions of bipolar disorder and schizophrenia. Molecular Psychiatry. 290 2013;19(9):1017-1024. doi:10.1038/mp.2013.138.

[10] Socrates A, Bond T, Karhunen V, Auvinen J, Rietveld C, Veijola J, et al. Polygenic risk scores ${ }^{292}$ applied to a single cohort reveal pleiotropy among hundreds of human phenotypes. bioRxiv. 2017;. 293 
[11] Power RA, Steinberg S, Bjornsdottir G, Rietveld CA, Abdellaoui A, Nivard MM, et al. Poly- 294 genic risk scores for schizophrenia and bipolar disorder predict creativity. Nature Neuroscience. 295 2015;18(7):953-955. doi:10.1038/nn.4040.

[12] Plomin R, von Stumm S. The new genetics of intelligence. Nature Reviews Genetics. ${ }^{297}$ 2018;doi:10.1038/nrg.2017.104.

[13] Hagenaars SP, Harris SE, Davies G, Hill WD, Liewald DCM, Ritchie SJ, et al. Shared genetic ${ }^{299}$ aetiology between cognitive functions and physical and mental health in UK Biobank $(\mathrm{N}=112151) \quad 300$ and 24 GWAS consortia. Molecular Psychiatry. 2016;21(11):1624-1632. doi:10.1038/mp.2015.225. 301

[14] Ripke S, Neale BM, Corvin A, Walters JTR, Farh KH, Holmans PA, et al. Biological insights from 302 108 schizophrenia-associated genetic loci. Nature. 2014;511:421-427. doi:10.1038/nature13595. 303

[15] Chatterjee N, Shi J, García-Closas M. Developing and evaluating polygenic risk prediction models 304 for stratified disease prevention. Nature Reviews Genetics. 2016;doi:10.1038/nrg.2016.27. 305

[16] Tremblay J, Hamet P. Role of genomics on the path to personalized medicine. Metabolism: clinical 306 and experimental. 2013;62 Suppl 1:S2-5. doi:10.1016/j.metabol.2012.08.023. 307

[17] Lenfant C. Prospects of personalized medicine in cardiovascular diseases. Metabolism: clinical and 308 experimental. 2013;62 Suppl 1:S6-10. doi:10.1016/j.metabol.2012.08.018.

[18] Chatterjee N, Wheeler B, Sampson J, Hartge P, Chanock SJ, Park JHH. Projecting the performance 310 of risk prediction based on polygenic analyses of genome-wide association studies. Nature Genetics. 311 2013;45(4):400-5, 405e1-3. doi:10.1038/ng.2579.

[19] Wray NR, Lee SH, Mehta D, Vinkhuyzen AAE, Dudbridge F, Middeldorp CM. Research Review: 313 Polygenic methods and their application to psychiatric traits. Journal of Child Psychology and 314 Psychiatry. 2014;55(10):1068-1087. doi:10.1111/jcpp.12295.

[20] Domingue BW, Belsky DW, Harris KM, Smolen A, McQueen MB, Boardman JD. Polygenic 316 risk predicts obesity in both white and black young adults. PloS one. 2014;9(7):e101596. 317 doi:10.1371/journal.pone.0101596.

[21] Tesli M, Espeseth T, Bettella F, Mattingsdal M, Aas M, Melle I, et al. Polygenic risk score 319 and the psychosis continuum model. Acta Psychiatrica Scandinavica. 2014;130(4):311-317. 320 doi:10.1111/acps.12307.

[22] Chang SC, Glymour MM, Walter S, Liang L, Koenen KC, Tchetgen EJ, et al. Genome-wide 322 polygenic scoring for a 14-year long-term average depression phenotype. Brain and behavior. 323 2014;4(2):298-311. doi:10.1002/brb3.205. 
[23] Machiela MJ, Chen CY, Chen C, Chanock SJ, Hunter DJ, Kraft P. Evaluation of polygenic risk 325 scores for predicting breast and prostate cancer risk. Genetic Epidemiology. 2011;35(6):506-514. 326 doi:10.1002/gepi.20600.

[24] Wray NR, Yang J, Hayes BJ, Price AL, Goddard ME, Visscher PM. Pitfalls of predicting complex 328 traits from SNPs. Nature reviews Genetics. 2013;14(7):507-15. doi:10.1038/nrg3457.

[25] Bycroft C, Freeman C, Petkova D, Band G, Delaneau O, Connell JO, et al. Genome-wide genetic 330 data on 500,000 UK Biobank participants. bioRxiv. 2017;doi:http://dx.doi.org/10.1101/166298. 331

[26] Diogo D, Tian C, Franklin C, Alanne-Kinnunen M, March M, Spencer C, et al. Phenome-wide 332 association studies (PheWAS) across large "real-world data" population cohorts support drug target 333 validation. bioRxiv. 2017; p. 1-37.

[27] Nielsen JB, Thorolfsdottir RB, Fritsche LG, Zhou W, Skov MW, Graham SE, et al. Genome-wide 335 association study of 1 million people identifies 111 loci for atrial fibrillation. bioRxiv. 2018;. 336

[28] Liu JZ, Erlich Y, Pickrell JK. Case-control association mapping by proxy using family history of 337 disease. Nature Genetics. 2017;49(3):325-331. doi:10.1038/ng.3766.

[29] Price AL, Patterson NJ, Plenge RM, Weinblatt ME, Shadick Na, Reich D. Principal compo- 339 nents analysis corrects for stratification in genome-wide association studies. Nature genetics. 340 2006;38(8):904-9. doi:10.1038/ng1847.

[30] Pearl J. Causality: Models, Reasoning, and Inference. New York: Cambridge University Press; 342 2000.

[31] Varma S, Simon R. Bias in error estimation when using cross-validation for model selection. BMC 344 Bioinformatics. 2006;7(1):91. doi:10.1186/1471-2105-7-91.

[32] Abraham G, Kowalczyk A, Zobel J, Inouye M. Performance and robustness of penalized and 346 unpenalized methods for genetic prediction of complex human disease. Genetic epidemiology. 347 2013;37(2):184-95. doi:10.1002/gepi.21698.

[33] de Maturana EL, Chanok SJ, Picornell AC, Rothman N, Herranz J, Calle ML, et al. Whole genome 349 prediction of bladder cancer risk with the Bayesian LASSO. Genetic epidemiology. 2014;38(5):467- 350 76. doi:10.1002/gepi.21809.

[34] Stahl E, Forstner A, McQuillin A, Ripke S, Ophoff R, Scott L, et al. Genomewide association study 352 identifies 30 loci associated with bipolar disorder. bioRxiv. 2017;. 
[35] Euesden J, Lewis CM, O’Reilly PF. PRSice: Polygenic Risk Score software. Bioinformatics. 354 2015;31(9):1466-1468. doi:10.1093/bioinformatics/btu848.

[36] Wood AR, Esko T, Yang J, Vedantam S, Pers TH, Gustafsson S, et al. Defining the role of common 356 variation in the genomic and biological architecture of adult human height. Nature Genetics. 357 2014;46(11):1173-1186. doi:10.1038/ng.3097.

[37] Locke AE, Kahali B, Berndt SI, Justice AE, Pers TH, Day FR, et al. Genetic studies 359 of body mass index yield new insights for obesity biology. Nature. 2015;518(7538):197-206. 360 doi:10.1038/nature14177.

[38] de Moor MHM, van den Berg SM, Verweij KJH, Krueger RF, Luciano M, Arias Vasquez 362 A, et al. Meta-analysis of Genome-wide Association Studies for Neuroticism, and the 363 Polygenic Association With Major Depressive Disorder. JAMA Psychiatry. 2015;72(7):642. 364 doi:10.1001/jamapsychiatry.2015.0554.

[39] Nikpay M, Goel A, Won HH, Hall LM, Willenborg C, Kanoni S, et al. A comprehensive 1,000 366 Genomes-based genome-wide association meta-analysis of coronary artery disease. Nature genetics. 367 2015;47(10):1121-30. doi:10.1038/ng.3396.

[40] Willer CJ, Schmidt EM, Sengupta S, Peloso GM, Gustafsson S, Kanoni S, et al. Discovery 369 and refinement of loci associated with lipid levels. Nature Genetics. 2013;45(11):1274-1285. 370 doi:10.1038/ng.2797.

[41] Mahajan A, Go MJ, Zhang W, Below JE, Gaulton KJ, Ferreira T, et al. Genome-wide trans-ancestry 372 meta-analysis provides insight into the genetic architecture of type 2 diabetes susceptibility. Nature 373 genetics. 2014;46(3):234-44. doi:10.1038/ng.2897.

[42] Chang CC, Chow CC, Tellier LC, Vattikuti S, Purcell SM, Lee JJ. Second-generation PLINK: rising 375 to the challenge of larger and richer datasets. GigaScience. 2015;4(1):1-16. doi:10.1186/s13742-015- 376 0047-8.

[43] Mak TSH, Porsch RM, Choi SW, Zhou X, Sham PC. Polygenic scores via penalized regression on 378 summary statistics. Genetic Epidemiology. 2017;(February):1-12. doi:10.1002/gepi.22050. 